\title{
Factores de riesgo que afectan la fusión en el tratamiento de la inestabilidad en la columna lumbar
}

\section{Fatores de risco que afetam a fusão no tratamento da instabilidade na coluna lombar}

\author{
Risk factors affecting the fusion in the lumbar spine instability \\ treatment
}

\author{
José María Jiménez Ávila' \\ Jorge García Valencia ${ }^{2}$ \\ Wadih Emilio Bitar Alatorre ${ }^{3}$
}

\section{RESUMEN}

Objetivo: describir cuales son los factores de riesgo que afectan la fusión vertebral en la inestabilidad de la columna lumbar, en pacientes atendidos en nuestro hospital relacionados con los aspectos nutricionales. Métodos: se revisaron 59 pacientes de los cuales $15(25,4 \%)$ fueron casos (pseudoartrosis) y 44 (74,6\%) fueron controles (fusión). La distribución demográfica presentó pacientes, de los cuales 27 $(45,8 \%)$ correspondieron al sexo femenino y $32(54,2 \%)$ al sexo masculino. El promedio de edad fue de de 51 con una desviacion standard de 12, una minima de 20 y una máxima de 85 años. Su distribución por diagnostico fue 29 (49,1\%) espondilolistesis; 21 $(35,6 \%)$ fracturas; 5 (8,5\%) hernias discales; 3 (5,1\%) infecciones y 1 $(1,7 \%)$ tumoración. El peso promedio fue de $75,9 \mathrm{~kg}$, con una talla de 1,60 cm, el IMC de 24,5, una Hb de 15,2, Hto de 35,9, y albúmina de 3,5. Resultados: se analizaron diversos factores de riesgo, entre la edad de mayores de

\section{RESUMO}

Objetivo: descrever quais são os fatores de risco que afetam a fusão vertebral na instabilidade da coluna lombar, de pacientes atendidos em nosso hospital que são relacionados com aspectos nutricionais. Métodos: foram revisados 59 pacientes dos quais $15(25,4 \%)$ foram casos (pseudoartrose) e $44(76,4 \%)$ foram controles (fusão). A distribuição demográfica apresentou pacientes e mostrou que $27(45,8 \%)$ eram do sexo feminino e $32(54,2 \%)$ do sexo masculino, com uma média de $51 \pm 12$, uma mínima de 20 e uma máxima de 85 anos. A sua distribuição por diagnóstico foi $29(49,1 \%)$ espondilolistese, $21(35,6 \%)$ fraturas, $5(8,5 \%)$ hérnias discais, $3(5,1 \%)$ infecções e $1(1,7 \%)$ tumor. O peso médio foi de $75,9 \mathrm{~kg}$, com uma altura de $1,60 \mathrm{~cm}$; IMC de $24,5, \mathrm{Hb}$ de 15,2 , Hto de 35,9 e albumina de 3,5 . Resultados: foram analisados diversos fatores de risco em maiores de 60 anos, o que mostrou $49 \%$ a mais de

\begin{abstract}
Objective: to describe the risk factors that affect the vertebral fusion in the lumbar spine instability, in patients attended in our hospital that are related to nutritional aspects. Methods: A total of 59 patients were reviewed of whom 15 (25.4\%) were cases (pseudoartrosis), and 44 (74.6\%) were controls (fusion). The
\end{abstract} population distribution presented patients, of which 27 (45.8\%) were female and $32(54.2 \%)$, male. The average age was 51 with a 12 standard deviation, a minimum of 20 and a maximum of 85 years old. Distribution by diagnosis was 29 (49.1\%) spondylolisthesis, $21(35.6 \%)$ fractures, 5 (8.5\%) herniations, 3 (5.1\%) infections, and 1 (1.7\%) tumor. The average weight was $75.9 \mathrm{~kg}$, with $1.60 \mathrm{~cm}$ height, BMI of 24.5, a $\mathrm{Hb}$ of 15.2, Hto of 35.9, and 3.5 albumin. Results: several risk factors were analyzed between ages higher

\footnotetext{
Trabajo realizado en Unidad Médica de Alta Especialidad del Centro Médico Nacional de Occidente "Lic. Ignacio García Téllez" - Guadalajara, Jalisco, México.

'Cirujano Ortopedista adscrito al Servicio de Ortopedia, Clínica de Columna del Centro Médico Nacional de Occidente, Unidad Médica de Alta Especialidad del Instituto Mexicano del Seguro Social - IMSS - Guadalajara, Jalisco, México.

${ }^{2}$ Residente del cuarto año adscrito al Servicio de Traumatología y Ortopedia del Centro Médico Nacional de Occidente, Unidad Médica de Alta Especialidad del Instituto Mexicano del Seguro Social - IMSS - Guadalajara, Jalisco, México.

${ }^{3} \mathrm{PhD}$, Jefe de División III Musculo-esquelético del Centro Médico Nacional de Occidente, Unidad Médica de Alta Especialidad del Instituto Mexicano del Seguro
} Social - IMSS - Guadalajara, Jalisco, México. 
60 años, se mostró un 49\% más probabilidad de una pseudoartrosis en comparación con el Grupo Control con un IC 95\%=0,12-2,27. El peso mayor de $90 \mathrm{~kg}$ mostró un 82\% más probabilidad de una pseudoartrosis en comparación con el Grupo de Fusión con un IC 95\%=0,03-0,43, el IMC muestra un 25\% más probabilidad de una pseudoartrosis con un IC 95\%=0,183,18 , la hipoalbuminemia menor de 3,4 mg/100 mL mostró un 78\% más probabilidad de la pseudoartrosis, con IC $95 \%=0,06-0,75$. Conclusiones: se encontró que la pseudoartrosis es provocada por factores de riesgo asociados al perfil nutricional como lo es la propia obesidad, la hipoalbuminemia y existe una probabilidad de presentarla de 1,5 veces más, que en las personas con valores normales. Por tal motivo, es importante que dichos valores sean evaluados y corregidos de manera prematura en el momento prequirúrgico, con medidas de soporte que permitan un adecuado control metabólico y, por consecuencia, un resultado óptimo que es la fusión. Nivel de evidencia: estudio de casos y controles con un nivel de evidencia III. probabilidade de uma pseudoartrose em comparação com o Grupo Controle, com IC 95\%=0,12-2,27, assim como o peso maior de $90 \mathrm{~kg}$, que mostrou $82 \%$ a mais de probabilidade de uma pseudoartrose em comparação com o Grupo de Fusão com IC $95 \%=0,03-0,43$. O IMC mostrou $25 \%$ a mais de probabilidade de uma pseudoartrose com IC $95 \%=0,18$ 3,18 , assim como a hipoalbuminemia menor que 3,4 mg/100 mL mostrando $78 \%$ a mais de probabilidade da pseudoartrose (IC 95\% $=0,06$ 0,75). Conclusões: encontrou-se que a pseudoartrose é provocada por fatores de risco associados ao perfil nutricional como a própria obesidade, a hipoalbuminemia, com uma probabilidade de 1,5 vezes a mais que as pessoas com valores normais. Por tal motivo, é importante que tais valores sejam avaliados e corrigidos anteriormente ao momento pré-cirúrgico, com medidas de apoio que permitam um adequado controle metabólico e, consequentemente, um ótimo resultado na fusão. Nível de evidência: estudo de casos e controles com nível de evidência III. than 60 years, it showed a 49\% more likely to a pseudoartrosis in comparison with the Control Group with a 95\% $C I=0.12-2.27$. More than $90 \mathrm{~kg}$ of weight showed $82 \%$ more likely to a pseudoartrosis in comparison with the Fusion Group, having as a 95\% CI=0.03-0.43, the BMI shows 25\% more likely to a pseudoartrosis with a $95 \% \mathrm{CI}=0.18$ 3.18, hypoalbuminemia less than $3.4 \mathrm{mg} / 100 \mathrm{~mL}$ showed a $78 \%$ more likely to the pseudoartrosis (95\% $C I=0.06-0.75)$. Conclusions: the pseudoartrosis is caused by risk factors associated with the nutrient profile, like the obesity itself, the hypoalbuminemia and there is a probability to present 1.5 times more than the values of ordinary people. Thus, it is important that these values are evaluated and fixed before in the pre-surgical with support measures that allows adequate metabolic and, consequently, an optimal outcome, such as fusion. Evidence level: study case-control with a level III of evidence.
DESCRIPTORES: Inestabilidad de la articulación/cirugía; Vértebras lumbares/cirugía; Factores de riesgo; Fusión vertebral/efectos adversos
DESCRITORES: Instabilidade articular/cirurgia; Vértebras lombares/cirurgia; Fatores de risco; Fusão vertebral/efeitos adversos
KEYWORDS: Joint instability/ surgery; Lumbar vertebrae/ surgery; Risk factors; Spinal fusion/adverse effects

\section{INTRODUCCIÓN}

Los factores de riesgo se definen como los endógenos o exógenos, que preceden al comienzo de la lesión y están asociados a un incremento de la probabilidad de incidencia de una enfermedad, en este caso específicamente la presencia de una pseudoartrosis posterior a una cirugía de columna ${ }^{1}$.

Su importancia radica, en que pueden ser controlados y que una vez que se modifican pueden cambiar el curso del resultado, previniendo los efectos adversos que provocan las complicaciones en los ya difíciles procedimientos de la cirugía espinal.

Una buena alimentación es parte de un efectivo tratamiento para todo paciente antes y después del procedimiento quirúrgico ${ }^{2}$, se ha demostrado que el estado nutricional tiene un impacto sobre los resultados clínicos de los procedimientos en general $^{3} \mathrm{y}$ en la fusión ósea, en forma específica ${ }^{4}$.

El estado nutricional del paciente previo a cualquier evento quirúrgico de columna que requiera fusión, se puede identificarlo mediante estudios de laboratorio. Por lo tanto, se puede monitorizar al paciente con indicadores como el índice de masa corporal, los linfocitos y la albúmina sérica ${ }^{2,5}$.

Aspectos como la perdida reciente de peso y las concentraciones séricas de albúmina menores de $3,4 \mathrm{mg} / 100$ $\mathrm{mL}$ deben ser señales importantes que indiquen la necesidad de valoración nutricional cuidadosa y la posible necesidad de apoyo nutricional ${ }^{3}$. Lenke et al. comprobaron que los pacientes que se someten a procedimientos de fusión raquídea pueden requerir de 6 a 12 semanas para recuperarse de la lesión nutricional perioperatoria y sugieren la 
necesidad de una valoración nutricional más enérgica en estos $\operatorname{casos}^{2,5}$.

El objetivo del presente trabajo es describir cuales son los factores de riesgo relacionados con los aspectos nutricionales que afectan la fusión vertebral y cuál es la fuerza de asociación que guardan con la pseudoartrosis, en la inestabilidad de la columna lumbar.

El protocolo ha sido aprobado previamente por un comité examinador ético y científico institucional en el sitio donde se efectúo el estudio.

\section{MÉTODOS}

Se realizó un diseño tipo casos y controles, con pacientes atendidos en la Unidad Médica de Alta Especialidad del Centro Médico Nacional de Occidente "Lic. Ignacio García Téllez”, en Guadalajara, Jalisco.

Se elaboró una hoja de captura la que contenía los datos de la ficha clínica, así como los factores de riesgo, mismos que fueron tomados del expediente clínico, agrupándose en dos categorías y tomando en cuenta los criterios de inclusión y siendo inidentificados como uno. Pseudoartrosis (Ausencia de consolidación ósea y presencia de zona fibrosa en el segmento afectado, una vez transcurrido el tiempo normal para la osificación en promedio más de 12 meses, y 2; Fusión. (Unión ósea entre dos espacios vertebrales después de una manipulación quirúrgica).

\section{MÉTODOS ESTADÍSTICOS}

Los datos fueron analizados usando un paquete de software comercial (SPSS para Windows, versión 10.0), las variables fueron analizadas mediante tablas de $2 \times 2$ para determinar el Odds Ratio y la significancia estadística mediante la prueba de Mantel-Haenszel, con un intervalo de confianza del $95 \%$. Un valor p menor de 0,05 se consideró como significativo.

\section{RESULTADOS}

Del periodo comprendido de Enero de 2005 a Diciembre de 2007, se estudiaron un total de 59 pacientes del Hospital de Especialidades del Centro Médico Nacional de Occidente, en Guadalajara, Jalisco, los que fueron tratados quirúrgicamente mediante una instrumentación en columna lumbar siempre y cuando cumplieran con los criterios de inclusión.

Los datos demográficos mostraron que 27 (45,8\%) pacientes fueron del sexo femenino y $32(54,2 \%)$ del sexo masculino, con una edad promedio de 51 años y una desviacion standard (d.s.) de 12, con un peso en promedio de $75 \mathrm{~kg}$, con una d.s. de 13, una talla de 1,60 cm, con una d.s. de $10 \mathrm{~cm}$. Fue diagnosticado espondilolistesis en 29 $(49,2 \%)$ pacientes, fracturas de la columna lumbar en 21 $(35,6 \%)$, hernia discal en $5(8,5 \%)$, mal de Pott en $3(5,1 \%)$ y espondilitis por Brucela en $1(1,7 \%)$.

En cuanto a las condiciones físicas, $27(45,8 \%)$ presentaban obesidad y $32(54,2 \%)$ su peso se encontraba de acuerdo a su talla; $3(5,1 \%)$ pacientes usaron algún tipo de corticoide y $56(94,9 \%)$ no lo usaron; 34 (56,6\%) tomaron alcohol y $25(42,4 \%)$ no lo hicieron; en cuanto al tabaquismo, $26(44,1 \%)$ fueron positivos y $33(55,9 \%)$ fueron negativos, el uso de drogas ocurrió en $3(5,1 \%)$ y $56(94,9 \%)$ fueron negativos, la calidad ósea en $51(86,4 \%)$ se reportó como normal y solo en $8(13,6 \%)$ había datos de osteopenia (Tabla 1).

Una vez recabados los datos demográficos, los pacientes fueron clasificados de acuerdo a los criterios de inclusión como caso, aquellos que presentaron una pseudoartrosis de los cuales fueron $15(25,4 \%)$ y una fusión normal en $44(74,6 \%)$.

De los estudios de laboratorio, se reportó en promedio una $\mathrm{Hb}$ de 12,6 con una d.s. de 2,5; Hto. de 35,9 con una d.s. de 8,7; linfocitos de 23.500 con una d.s. de 13.200; glicemia de 104,2 con una d.s. de 34,4 y una albumina en promedio de 3,1 con una d.s. de 0,5 (Tabla 1).

Se analizaron diversos factores de riesgo entre los que destaca la edad, misma que se tomó como referencia las personas mayores de 60 años, lo cual mostró un riesgo de siete veces mayor probabilidad de una pseudoartrosis en comparación con el Grupo Control con un IC 95\% de 1,8035,64 . La obesidad mostró una probabilidad del 5\% mayor de presentar una pseudoartrosis en comparación con el Grupo de Fusión con un IC 95\% de 0,28-3,95, el índice de masa corporal (IMC) muestra un OR de 0,97 con un IC 95\% de 0,12-6,51, la hipoalbuminemia menor de 3,4 $\mathrm{mg} / 100 \mathrm{~mL}$ mostró un $82 \%$ más probabilidad de presentar la pseudoartrosis, (IC $95 \%=0.48-6.95$ ) y la linfopenia $(<1,500)$ mostró un $63 \%$ mayor probabilidad de que los pacientes presentaran una pseudoartrosis en comparación con los pacientes con albumina por arriba de $3,5 \mathrm{mg} / 100$ $\mathrm{mL}$ con un IC $95 \%$ de $0,37-5,19$. La densidad ósea apresentó una probabilidad del $67 \%$ mayor con un IC $95 \%$ de 0,31-5,53 de una pseudoartrosis en comparación con el Grupo Control, y otro factor asociado que se valoro fue el tabaquismo, lo cual arrojó un OR de 0,55 con un IC $95 \%$ de 0,13 a 2,5 (Tabla 2).

\section{DISCUSIÓN}

En el presente estudio, es posible observar que la pseudoartrosis es provocada por factores de riesgo múltiples, y algunos de ellos se encuentran asociados al perfil nutricional. Lo más importante es que pueden ser detectados desde la consulta externa, lo que en algunos casos la probabilidad de una pseudoartrosis ser prevenible, tal es el caso de la propia obesidad, acompañada de una valoración especifica del IMC, la hipoalbuminemia y la linfopenia ${ }^{6-9}$.

La obesidad como factor único puede incrementar un $5 \%$ la probabilidad de una pseudoartrosis, que si le agregamos factores como la hipoalbuminemia, este se puede incrementar hasta un $42 \%$ la probabilidad de un resultado no deseado $8,10,11$.

La densidad ósea también nos marca una probabilidad del $33 \%$ y la edad mayor de 60 años puede llegar a ser siete 
TABLA 1 - Datos demográficos

\begin{tabular}{|c|c|c|c|c|c|c|}
\hline Variables & & $\begin{array}{l}\text { Frecuencia } \\
\text { absoluta }\end{array}$ & $\begin{array}{l}\text { Frecuencia } \\
\text { relativa \% }\end{array}$ & Variables & Promedio & \\
\hline \multirow[t]{2}{*}{ Sexo } & Femenino & 27 & 45,8 & Edad & 51,1 & 12,8 \\
\hline & Masculino & 32 & 54,2 & Peso & 75,9 & 13,9 \\
\hline \multirow[t]{2}{*}{ Obesidad } & $\mathrm{Si}$ & 27 & 45,8 & Talla & 1,6 & 0,1 \\
\hline & No & 32 & 54,2 & Evolución & 3,5 & 1,4 \\
\hline \multirow[t]{2}{*}{ Corticoterapia } & $\mathrm{Si}$ & 3 & 5,1 & Tpo Qx & 3,3 & 0,8 \\
\hline & No & 56 & 94,9 & IMC & 24,5 & 2,1 \\
\hline \multirow[t]{2}{*}{ Alcoholismo } & $\mathrm{Si}$ & 34 & 56,6 & $\mathrm{Hb}$ & 12,6 & 2,5 \\
\hline & No & 25 & 42,4 & Hto & 35,9 & 8,7 \\
\hline \multirow[t]{2}{*}{ Tabaquismo } & $\mathrm{Si}$ & 26 & 44,1 & Linfocitos & 23,5 & 13,2 \\
\hline & No & 33 & 55,9 & Albumina & 3,1 & 0,5 \\
\hline \multirow[t]{2}{*}{ Drogas } & $\mathrm{Si}$ & 3 & 5,1 & Glucosa & 104,2 & 34,4 \\
\hline & No & 56 & 94,9 & & & \\
\hline \multirow[t]{2}{*}{ Densidad ósea } & Normal & 51 & 86,4 & & & \\
\hline & Osteopenia & 8 & 13,6 & & & \\
\hline \multirow[t]{2}{*}{ Resultado } & Pseudoartrosis & 15 & 25,4 & & & \\
\hline & Fusión & 44 & 74,6 & & & \\
\hline \multirow[t]{2}{*}{ IMC } & $<24,9$ & 17 & 28,8 & & & \\
\hline & $>25$ & 42 & 71,2 & & & \\
\hline \multirow[t]{5}{*}{ Diagnostico } & Espondilolistesis & 29 & 49,2 & & & \\
\hline & Fracturas & 21 & 35,6 & & & \\
\hline & Hernia discal & 5 & 8,5 & & & \\
\hline & Mal de Pott & 3 & 5,1 & & & \\
\hline & Espon. por Brucela & 1 & 1,7 & & & \\
\hline
\end{tabular}

TABLA 2 - Distribución de pseudoartrosis y factores coadyuvantes negativos que afectan la fusión

\begin{tabular}{|c|c|c|c|c|c|c|}
\hline Variables & & $\%$ & & $\%$ & OR & IC 95\% \\
\hline Edad & $>60$ & & $<59$ & & & \\
\hline Pseudoartrosis & 10 & 16,9 & 5 & 8,5 & 7,78 & $1,8-35,64$ \\
\hline Fusión & 9 & 15,3 & 35 & 59,3 & 1 & \\
\hline Obesidad & $>90$ & & $<90$ & & & \\
\hline Pseudoartrosis & 7 & 11,8 & 8 & 13,7 & 1,05 & $0,28-3,95$ \\
\hline Fusión & 20 & 33,9 & 24 & 40,6 & 1 & \\
\hline IMC & $>25$ & & $<24,9$ & & & \\
\hline Pseudoartrosis & 2 & 3,3 & 13 & 22 & 0,97 & $0,12-6,51$ \\
\hline Fusión & 6 & 10,2 & 38 & 64,5 & 1 & \\
\hline Albumina & $<3,5$ & & $>3,4$ & & & \\
\hline Pseudoartrosis & 8 & 13,6 & 7 & 11,8 & 1,37 & $0,37-5,19$ \\
\hline Fusión & 20 & 33,9 & 24 & 40,7 & 1 & \\
\hline Densidad ósea & Osteopenia & $\%$ & Normal & $\%$ & & \\
\hline Pseudoartrosis & 2 & 3,3 & 13 & 22 & 1,33 & $0,31-5,53$ \\
\hline Fusión & 6 & 10,1 & 38 & 64,4 & 1 & \\
\hline Tabaquismo & $\mathrm{Si}$ & & No & & & \\
\hline Pseudoartrosis & 5 & 8,5 & 10 & 16,9 & 0,55 & $0,13-2,15$ \\
\hline Fusión & 21 & 35,7 & 23 & 38,9 & 1 & \\
\hline Hipoalbuminemia & $<3,5$ & & $>3,6$ & & & \\
\hline Pseudoartrosis & 8 & 13,5 & 7 & 11,9 & 1,82 & $0,48-6,95$ \\
\hline Fusión & 17 & 28,8 & 27 & 45,8 & 1 & \\
\hline Linfopenia & $<1,500$ & & $>1,501$ & & & \\
\hline Pseudoartrosis & 8 & 13,6 & 7 & 11,8 & 1,37 & $0,37-5,19$ \\
\hline Fusión & 20 & 33,9 & 24 & 40,7 & 1 & \\
\hline
\end{tabular}


veces mayor la probabilidad de que no exista una fusión en la zona de la cirugía $a^{7,12,13}$.

Cabe destacar que todos estos factores al unirse y encontrarse presentes en un solo paciente, el riesgo puede ser de tres a cuatro veces mayor, por lo que es importante que desde la consulta detectemos estos valores, ya que muchos exámenes, como la albumina sérica, no son un estudio que se realice de rutina para planear la cirugía de un paciente.

Por tal motivo, es importante que dichos valores sean evaluados y también corregidos de manera prematura, con medidas de soporte que permitan un adecuado control metabólico y por consecuencia para obter un resultado óptimo como lo es la fusión ${ }^{14-16}$.
Por lo tanto, el estado nutricional si afecta la evolución satisfactoria en los pacientes sometidos a estabilización en cirugía de columna lumbar ${ }^{8,10,14}$.

Todo esto debe de estar acompañado de una verdadera comunicación con el paciente y que este se informe de los probables riesgos de un resultado no deseado, así como algunos otros factores como lo es el tabaquismo, logrando su comprensión para que al final, el paciente decida si desea operarse, dándole la fortaleza al documento denominado como consentimiento informado, el cual debe de contener de manera objetiva esas probabilidades de riesgo que el paciente tiene ante su situación nutricional ${ }^{8,9}$.

\section{REFERÊNCIAS}

1. Grisso JA, Kelsey JL, O'Brien LA, Miles CG, Sidney S, Maislin G, et al. Risk factors for hip fracture in men. Am J Epidemiol. 1997;145(9):786-93.

2. Lenke LG, Bridwell KH, Blanke K, Baldus C. Prospective analysis of nutritional status normalization after spinal reconstructive surgery. Spine (Phila Pa 1976). 1995;20(12):1359-67.

3. Dickhaut SC, DeLee JC, Page CP. Nutritional status: importance in predicting wound-healing after amputation. J Bone Joint Surg Am. 1984;66(1):71-5.

4. Zdeblick TA. A prospective, randomized study of lumbar fusion. Preliminary results. Spine (Phila $\mathrm{Pa}$ 1976). 1993;18(8):983-91.

5. Lehman RA Jr, Lenke LG. Longsegment fusion for the thoracolumbar spine in conjunction with a motionpreserving artificial disc replacement: case report and review of the literature. Spine (Phila Pa 1976). 2007;32(7):E240-5.

6. Daniels AH, Arthur M, Hart RA. Variability in rates of arthrodesis for patients with thoracolumbar spine fractures with and without associated neurologic injury. Spine (Phila $\mathrm{Pa}$ 1976). 2007;32(21):2334-8.

7. DiPaola CP, Molinari RW. Posterior lumbar interbody fusion. J Am Acad Orthop Surg. 2008;16(3):130-9.
8. Einhorn TA, Bonnarens F, Burstein $\mathrm{AH}$. The contributions of dietary protein and mineral to the healing of experimental fractures. A biomechanical study. J Bone Joint Surg Am. 1986;68(9):1389-95.

9. Jensen JE, Jensen TG, Smith TK, Johnston DA, Dudrick SJ. Nutrition in orthopaedic surgery. J Bone Joint Surg Am. 1982;64(9):1263-72.

10.Martin BI, Mirza SK, Comstock BA, Gray DT, Kreuter W, Deyo RA. Reoperation rates following lumbar spine surgery and the influence of spinal fusion procedures. Spine (Phila Pa 1976). 2007;32(3):382-7.

11. Glaser J, Stanley M, Sayre H, Woody J, Found E, Spratt K. A 10-year follow-up evaluation of lumbar spine fusion with pedicle screw fixation. Spine (Phila Pa 1976). 2003; 28(13):1390-5.

12.Silcox DH 3rd, Daftari T, Boden SD, Schimandle JH, Hutton WC, Whitesides TE Jr. The effect of nicotine on spinal fusion. Spine (Phila Pa 1976). 1995;20(14):1549-53.

13.Stevenson JC, Lees B, Devenport M, Cust MP, Ganger KF. Determinants of bone density in normal women: risk factors for future osteoporosis? BMJ. 1989;298(6678):924-8.
14.Dai LY, Jiang LS. Single-level instrumented posterolateral fusion of lumbar spine with beta-tricalcium phosphate versus autograph: a prospective, randomized study with 3-year follow-up. Spine (Phila Pa 1976). 2008;33(12):1299-304.

15.McDonough PW, Davis R, Tribus $\mathrm{C}$, Zdeblick TA. The management of acute thoracolumbar burst fractures with anterior corpectomy and Z-plate fixation. Spine (Phila Pa 1976). 2004;29(17):1901-8; discussion 1909.

16.Yu WD, Bernstein RM, Watts HG. Autogenous tibial strut grafts used in anterior spinal fusion for severe kyphosis and kyphoscoliosis. Spine (Phila Pa 1976). 2003;28(7):699-705.

\author{
Correspondencia \\ José María Jiménez Ávila \\ Avenida Circunvalación Jorge Álvarez \\ del Castillo, 1.558 \\ Colonia Chapultepec Country. \\ CP: 44610 \\ Guadalaraja, México \\ Teléfono: 013313770618 \\ Fax: 013313770619 \\ E-mail: josemajimeneza@hotmail.com
}

